\title{
Detection of CK19, LUNX, and KS1/4 mRNA expression in the peripheral blood for diagnosis of micrometastases in patients with non-small cell lung cancer and their clinical implications
}

\author{
L. Yan ${ }^{1 *}$, Y. Yao ${ }^{2 *}$, L.H. Wang ${ }^{1}$, M.L. Wang ${ }^{1}$ and X.H. Fu ${ }^{1}$ \\ ${ }^{1}$ Department of Respiratory Diseases, \\ the Affiliated Hospital of the Inner Mongolia Medical University, Hohhot, China \\ ${ }^{2}$ School of Life Sciences, Inner Mongolia University, Hohhot, China \\ *These authors contributed equally to this study. \\ Corresponding author: X.H. Fu \\ E-mail: liufuxiuhua@163.com
}

Genet. Mol. Res. 14 (4): 15090-15095 (2015)

Received June 23, 2015

Accepted September 30, 2015

Published November 24, 2015

DOI http://dx.doi.org/10.4238/2015.November.24.17

\begin{abstract}
The expression of CK19, LUNX, and KS1/4 mRNA biomarkers was detected in the peripheral blood of non-small cell lung cancer (NSCLC) patients to investigate the feasibility of indicating lung cancer micrometastases. Micrometastases were identified in the peripheral blood of 32 NSCLC patients, 15 benign pulmonary disease (BPD) patients, and 10 healthy volunteers by reverse transcriptase-polymerase chain reaction. The detection rates of CK19, LUNX, and KS1/4 mRNA-positive cells in the peripheral blood obtained from the NSCLC group were $34.4 \%(11 / 32)$, $37.5 \%(12 / 32)$, and $25 \%(8 / 32)$, respectively. CK19, LUNX, and KS1/4 mRNA-positive cells were detected in $6.6 \%(1 / 15), 0.0 \%(0 / 15)$, and $13.3 \%$ $(2 / 15)$ of the patients with BPD, respectively. However, the healthy group did not express any of the three markers. The expression of CK19, LUNX, and KS1/4 mRNA was significantly higher in the NSCLC group than that in the healthy and BPD groups $(P<0.05)$. CK19 and $L U N X$ mRNA may be ideal biomarkers indicating micrometastases in patients with NSCLC; however,
\end{abstract}


the diagnostic applicability of KS1/4 mRNA remains uncertain. The rate of expression of CK19 was not correlated with the clinicopathological characteristics $(P>0.05)$. The rate of expression of $L U N X$ and $K S 1 / 4$ was closely related to the clinical stage $(P<0.05)$, and not related to the clinical characteristics of the disease (age, gender, smoking history, pathological type, histologic classification, and differentiation; $P>0.05$ ).

Key words: Non-small cell lung cancer; Micrometastases; CK19 mRNA; LUNX mRNA; KS1/4 mRNA; RT-PCR

\section{INTRODUCTION}

Non-small-cell lung cancer (NSCLC) accounts for approximately $80 \%$ of the lung cancers (Peters et al., 2012). Surgery is the most common type of treatment for this type of cancer; however, the patients amenable to radical surgery have a 5-year overall survival rate of $50 \%$. NSCLC recurrence or metastasis may be a result of residual cancer cells in the peripheral blood. The identification of an ideal biomarker to indicate micrometastases, and the development of effective intervention strategies helps improve the prognosis of NSCLC patients. The application of reverse transcriptase polymerase chain reaction (RT-PCR) for the detection of micrometastases in the peripheral blood of NSCLC patients offers a readily accessible biological parameter that may be useful in monitoring disease progression. In order to establish the value of biomarker-based surveillance of micrometastases in NSCLC patients, it is necessary to correlate the presence of NSCLC cells (detected by RT-PCR) with established clinicopathological indicators.

In this study, RT-PCR was used to detect the expression of CK19, LUNX, and KS1/4 mRNA in the peripheral blood of NSCLC patients, and the correlation between these three mRNAbased biomarkers and the clinicopathological parameters of NSCLC patients was determined.

\section{MATERIAL AND METHODS}

\section{Subjects}

The control population consisted of 15 healthy volunteers with no clinical history of malignancy and 15 patients with benign pulmonary diseases (BPD). All patients included in the study had been newly diagnosed at our institution from February to November 2014. All patients underwent radical lobectomy and systematic lymph node dissection, but specifically denied any previous history of radiation or/and chemotherapy. The NSCLC group consisted of 32 patients (27 men, 5 women), aged between 45 and 70 years (mean age $58.9 \pm 7.1$ years). The study population consisted of 7 patients with low-differentiative NSCLC, 21 patients with moderatelydifferentiative NSCLC, and 4 patients with high-differentiative NSCLC. The lung cancer staging was revised based on the guidelines of the International Association for the Study of Lung Cancer (IASLC) system (Goldstraw and Crowley, 2006). Based on these criteria, 12, 11 and, 9 patients were classified into tumor stage I, II, and III, respectively.

\section{Collection of venous blood samples}

The study protocols were reviewed and approved by the institutional review board. 
Peripheral blood samples were obtained from all patients and controls with informed consent. To prevent contamination, only the second sequential $5 \mathrm{~mL}$ blood sample collected in an ethylenediamine-tetra acetic acid (EDTA)-containing tube was used for analysis. The blood samples were transported on ice to the laboratory for RNA isolation within $2 \mathrm{~h}$ of collection.

\section{Extraction of total RNA and reverse transcriptase PCR}

Total RNA was isolated from $1 \mathrm{~mL}$ of the blood sample using RNAiso Blood (TaKaRa, Otsu, Japan) according to the manufacturer protocols. All RNA extracts were analyzed by agarose gel electrophoresis and UV spectrophotometry. First strand cDNA synthesis was performed using the PrimeScript ${ }^{\mathrm{TM}} \mathrm{RT}$ Master Mix (Takara, Japan), according to protocols provided by the manufacturer. Specific CK19, LUNX, and KS1/4 cDNA sequences were amplified using the TaKaRa Taq ${ }^{\mathrm{TM}}$ Version 2.0 plus dye (TaKaRa) according to the manufacturer protocol. In brief, a $25 \mu \mathrm{L}$ reaction mixture, composed of $4 \mu \mathrm{L}$ cDNA product (equivalent to $200 \mathrm{ng}$ RNA), $12.5 \mu \mathrm{L} 2 \mathrm{X}$ Taq PCR Mix, $1 \mu \mathrm{L}$ specific primers $(10 \mathrm{mM})$, and $6.5 \mu \mathrm{L}$ RNA free $\mathrm{H}_{2} \mathrm{O}$, was used. The PCR was performed in aTProfessiona thermal cycler (Biometra, Germany), using the default cycling conditions ( 30 cycles of $95^{\circ} \mathrm{C}$ for $5 \mathrm{~s}$, $60^{\circ} \mathrm{C}$ for $30 \mathrm{~s}$, and $72^{\circ} \mathrm{C}$ for $30 \mathrm{~s}$, after an initial incubation at $95^{\circ} \mathrm{C}$ for $30 \mathrm{~s}$ ). Primers for 3 candidate genes were designed using the Primer Premier v 5.0 software program. The primer sequences are listed in Table 1. Expression values were normalized to that of the reference gene (GAPDH) gene. The experiments were repeated thrice, with 2 technical replicates for each experiment (Table 1).

\begin{tabular}{ll}
\multicolumn{2}{l}{ Table 1. Real time polymerase chain reaction (RT-PCR) primers. } \\
\hline Gene & Primer sequence \\
\hline CK19 & Forward: 5'-AGGTGGATTCCGCTCCGGGCA-3' \\
LUNX & Reverse: 5'-ATCTTCCTGTCCCTCGAGCA-3' \\
KS1/4 & Forward: 5'-CTCATTGTCTTCTACGGGCTGTTAG-3' \\
GAPDH & Reverse: 5'-CTTTATGCCGAGAGGGATGGT-3' \\
& Forward: 5'-CGCAGCTCAGGAAGAATGTG-3' \\
& Reverse: 5'-TGAATACACTGGCATTGACGA-3' \\
& Forward: 5'-CGGAGTCAACGGATTGGTCGTCGTAT-3' \\
& Reverse: 5'-AGCCTTCTCCATGGTGGTGAAGAC-3' \\
\hline
\end{tabular}

\section{Statistical analysis}

Clinical and pathological information was obtained from the clinical records of the patient. The relationship between the clinical and pathological parameters and the expression of the three mRNA-based biomarkers was initially explored by one-way analysis of variance (ANOVA), followed by the least significant difference multiple comparison test. All statistical analyses were performed on the SPSS (v.13.0) software platform for Windows (SPSS Statistics, IBM, Armonk, NY, USA). P values $<0.05$ were considered to be statistically significant.

\section{RESULTS}

\section{Calibration of CK19, LUNX and KS1/4 mRNA detection by RT-PCR}

The expression of CK19 mRNA, LUNX mRNA and KS1/4 mRNA was initially examined by RT-PCR in the peripheral blood of NSCLC, BPD, and healthy groups. CK19, LUNX, and KS1/4 
mRNA was detected $34.4 \%$ (11/32), 37.5\% (12/32), and $25 \%$ (8/32) of the peripheral blood samples extracted from 32 patients with NSCLC, respectively. The positive expression rate of CK19, LUNX, and $K S 1 / 4$ mRNA was $6.6 \%(1 / 15), 0.0 \%(0 / 15)$, and $13.3 \%$ (2/15), respectively, in the BPD group, while the positive expression rate of the three mRNA-based biomarkers was $0.0 \%$ in the healthy group. The positive expression rate of $C K 19, L U N X$, and $K S 1 / 4$ was found to be significantly higher in the NSCLC group than that in the BPD group $(P<0.05)$.

\section{Association between clinicopathological parameters and detection of CK19, LUNX, and KS1/4 mRNA in peripheral blood}

The correlation between the detection of CK19, LUNX, and KS1/4 mRNA in peripheral blood and the clinicopathological parameters of NSCLC patients is presented in Table 2. LUNX and $K S 1 / 4$ mRNA expression was closely related to the clinical stage of the disease; however, the mRNA expression of these two factors showed no correlation with the age, sex, smoking history, pathological type, histologic classification, and differentiation. On the other hand, CK19 mRNA expression was also not significantly correlated with the age, sex, smoking history, pathological type, histologic classification, differentiation, and clinical stage of the disease (Table 2).

\begin{tabular}{|c|c|c|c|c|c|c|c|}
\hline Characteristics & Number & CK19+ & $P$ value & LUNX + & $P$ value & KS1/4+ & $P$ value \\
\hline Age & & & 0.390 & & 0.647 & & 1.000 \\
\hline$\leq 50$ & 6 & 3 & & 6 & & 1 & \\
\hline$>50$ & 26 & 8 & & 26 & & 7 & \\
\hline Gender & & & 0.428 & & 0.626 & & 0.578 \\
\hline Male & 27 & 10 & & 27 & & 6 & \\
\hline Female & 5 & 1 & & 5 & & 2 & \\
\hline Smoking history & & & 0.465 & & 0.452 & & 0.433 \\
\hline Yes & 20 & 8 & & 9 & & 4 & \\
\hline No & 12 & 3 & & 3 & & 4 & \\
\hline Primary tumor & & & 0.703 & & 0.465 & & 1.000 \\
\hline $\mathrm{T} 1$ & 11 & 3 & & 3 & & 3 & \\
\hline $\mathrm{T} 2$ & 21 & 8 & & 9 & & 5 & \\
\hline Histological classification & & & 0.425 & & 0.248 & & 0.681 \\
\hline Adenocarcinoma & 10 & 2 & & 2 & & 3 & \\
\hline Squamous carcinoma & 22 & 9 & & 10 & & 5 & \\
\hline Differential & & & 0.374 & & 0.374 & & 0.646 \\
\hline Poor & 7 & 1 & & 1 & & 1 & \\
\hline Moderate + high & 25 & 10 & & 10 & & 7 & \\
\hline Stage & & & 0.681 & & 0.049 & & 0.001 \\
\hline$I+I I$ & 23 & 7 & & 6 & & 3 & \\
\hline III & 9 & 4 & & 6 & & 7 & \\
\hline
\end{tabular}

\section{DISCUSSION}

Micrometastases refers to the process with which non-haematological malignant cells spread and survive in the circulatory and lymphatic systems, bone marrow, and other organs, prior to the formation of metastatic nodules. Meanwhile, the development of micrometastases has no clinical manifestations, and it is therefore difficult to detect a single cell or cell colony using routine experimental methods (Coello et al., 2004). Ashworth discovered micrometastases in peripheral blood for the first time in 1869. Cancer cells enter the circulatory system; initially, a majority of the 
cancer cells are cleaned by the body's immune system when transferred into the circulatory system. As a result of this process, only a few cancer cells are kept alive; however, these cells find it difficult to metastasize (Ono et al., 2009). Micrometastases need not metastasize. However, if cancer cells are detected in the peripheral blood, it is likely to metastasize and recur. Previous studies have utilized members of the cytokeratin family (CKS) as biomarkers of micrometastases. CK19 is specifically expressed in epithelial tumor and normal epithelial cells. The presence ofCK19-positive cells in peripheral blood suggests the existence of cancer cells (Arrieta et al., 2014). In our study, CK19 mRNA was detected in $34.4 \%$ (11/32) of the peripheral blood samples obtained from NSCLC patients. This result suggested the transfer of cancer cells to the blood. However, false-positive cases have also been reported by some researchers. This may be related to contamination with skin cells at venipuncture (Dingemans et al., 1997). Strict precautions were employed in this study, and $1 \mathrm{~mL}$ original blood was discarded to avoid contamination with skin cells at venipuncture, in order to eliminate false-positive results. However, one CK19-positive patient was still found in the BPD group. This result may be attributed to tainted samples coming from inflammatory epithelial cells.

A previous report by Mitas et al identified $L U N X$ to be a sensitive biomarker of lung cancer micrometastases (by RT-PCR), compared to five other cancer-associated genes (muc1, KS1/4, CEA, CK19, and PSE) (Mitas et al., 2003b). LUNX has also been proved to be the most sensitive biomarker, with a high expression in hydrothorax and peripheral blood (Cheng et al., 2008). In our study, we observed that $37.5 \%(12 / 32)$ of the NSCLC patients displayed a positive expression of LUNX mRNA in the peripheral blood, which was higher than that of the other two mRNA. However, the expression of LUNX mRNA was not tested in healthy and BPD groups. Further study revealed that the biomarker LUNX was closely related to the clinical stage. Therefore, it may be a useful indicator for predicting micrometastases in NSCLC patients.

Wallace et al. (2005) analyzed the expression of LUNX, KS1/4, MUCI, CK19, and CEA mRNA in the lymphaden of NSCLC patients to indicate micrometastases; they concluded that 93 and $56 \%$ of the patients displaying micrometastases were KS1/4-positive LUNX-positive, respectively (Wallace et al., 2005). Another study observed a 100\% expression of KS1/4 in the mediastinal lymph nodes of patients with NSCLC (Mitas et al., 2003a). However, in our study, only $25 \%$ of the NCSLC patients were KS1/4-positive. In addition, KS1/4 mRNA was detected in the peripheral blood of $2 / 15(13.3 \%)$ of patients with BPD. Therefore, the expression of $K S 1 / 4$ mRNA must be tested in the peripheral blood in the future to indicate the presence of micrometastases in NSCLC patients.

In conclusion, the detection of LUNX mRNA expression in the peripheral blood of NSCLC patients was both highly sensitive and specific, and could therefore be a good biomarker to indicate micrometastases. The test for the expression of CK19 mRNA showed the highest sensitivity and low specificity. Moreover, the applicability of testing for the expression of KS1/4 mRNA remains to be determined.

As of today (April 15, 2015), the 32 NSCLC patients included in this study were non-shedding, with 2 relapsing during a follow-up examination. Therefore, the presence of micrometastases in the peripheral blood does not indicate eventual metastasis. A positive signal for mRNA biomarker could indicate that the cancer may have metastasized. Therefore, the patients must be subjected to aggressive treatment regimens to inhibit postoperative recurrence and metastasis. Moreover, dynamic testing may help clinicians individualize the treatment plans for NSCLC patients.

\section{Conflict of interest}

The authors declare no conflict of interest. 


\section{ACKNOWLEDGMENTS}

Research supported by grants from the Natural Science Foundation of Inner Mongolia (Grant \#2009MS1119 to Xiuhua Fu).

\section{REFERENCES}

Arrieta O, Pineda B, Muñiz-Hernández S, Flores D, et al. (2014). Molecular detection and prognostic value of epithelial markers mRNA expression in peripheral blood of advanced non-small cell lung cancer patients. Cancer Biomark. 14: 215-223.

Cheng M, Chen Y, Yu X, Tian Z, et al. (2008). Diagnostic utility of LunX mRNA in peripheral blood and pleural fluid in patients with primary non-small cell lung cancer. BMC Cancer 8: 156.

Coello MC, Luketich JD, Litle VR and Godfrey TE (2004). Prognostic significance of micrometastasis in non-small-cell lung cancer. Clin. Lung Cancer 5: 214-225.

Dingemans A, Brakenhoff RH, Postmus PE and Giaccone G (1997). Detection of cytokeratin-19 transcripts by reverse transcriptase-polymerase chain reaction in lung cancer cell lines and blood of lung cancer patients. Lab. Invest. 77 : 213-220.

Goldstraw P and Crowley JJ (2006). The international association for the study of lung cancer international staging project on lung cancer. J. Thorac. Oncol. 1: 281-286.

Mitas M, Cole DJ, Hoover L, Fraig MM, et al. (2003a). Real-time reverse transcription-PCR detects KS1/4 mRNA in mediastinal lymph nodes from patients with non-small cell lung cancer. Clin. Chem. 49: 312-315.

Mitas M, Hoover L, Silvestri G, Reed C, et al. (2003b). Lunx is a superior molecular marker for detection of non-small lung cell cancer in peripheral blood. J. Mol. Diagn. 5: 237-242.

Ono T, Minamiya Y, Ito M, Saito H, et al. (2009). Sentinel node mapping and micrometastasis in patients with clinical stage IA non-small cell lung cancer. Interact. Cardiovasc. Thorac. Surg. 9: 659-661.

Peters S, Adjei AA, Gridelli C, Reck M, et al. (2012). Metastatic non-small-cell lung cancer (NSCLC): ESMO clinical practice guidelines for diagnosis, treatment and follow-up. Ann. Oncol. 23: vii56-vii64.

Wallace MB, Block MI, Gillanders W, Ravenel J, et al. (2005). Accurate molecular detection of non-small cell lung cancer metastases in mediastinal lymph nodes sampled by endoscopic ultrasound-guided needle aspiration. Chest J. 127: 430437. 